\section{Corporate culture: protect idea factories}

It is unsurprising that universities have adopted corporate culture (Nature 540, 315; 2016), but surprising that they select such archaic models.

Universities corporatize because they must raise funds through teaching, research and commercialization. They need to publish research results openly, even though public funds don't cover full production costs.

Universities are the only social institutions set up specifically to produce ideas, and this is their most valuable societal role. Schools teach, governments collect data, firms patent inventions - and they all use ideas created by universities. Governments demand innovation, but reward income, not ideas.

Many universities have copied the manufacturing models of the 1950s. Power has shifted from academics to administrators. Academics are treated as interchangeable and replaceable, and performance is set through production quotas. Whereas academics judge peers by ideas, administrators count only cash. Knowledge enterprises such as Google and Facebook, by contrast, recognize that ideas need an encouraging and creative psychological environment.

Universities are idea factories. Current corporatization approaches emphasize the factory rather than the ideas. As governments curtail science, investing in ideas will be a winning strategy. Vicechancellors and education ministers, please listen. Ralf Buckley Griffith University, Australia.

r.buckley@griffith.edu.au

\section{Commercial hurdles to wave power}

The variable nature of wave power is the main reason why it is expensive and difficult to collect (see Z. L. Wang Nature 542, 159-160; 2017). Wavepower devices have much more demanding structural requirements than do offshore wind farms, for instance.

Contrary to Zhong Lin Wang's implication that wave energy is plentiful in all weathers, wave power fluctuates widely because it is created by accumulated wind stress. Monthly averages of wave power typically vary by a factor of six, compared with a factor of two for wind power. Daily variations can be even higher (B. Sørensen Renewable Energy; Academic, 2010).

I also question Wang's contention that "waves involve slow flows and oscillations in random directions". Wave motion is not random because it involves circular motions in the vertical plane, the direction of which is governed by earlier wind flows.

The high ratio of peak-toaverage power makes wave power difficult to commercialize. High costs for Wang's frictional nanogenerators and for pneumatic or mechanical wavepower devices are inevitable.

Bent Sørensen Roskilde University, Denmark. boson@ruc.dk

\section{Listen to accused Turkish scientists}

In my view, you misrepresent aspects of the problems confronting scientists in Turkey (Nature 542, 286-288; 2017). As a Turkish scientist working abroad, I contend that Turkey's government is using its former political ally, the Gülen movement, as a scapegoat to further its own ends.

For example, you suggest that the national researchfunding agency TÜBİTAK was "infiltrated" by supporters of the exiled preacher Fethullah Gülen. However, the accused are ordinary Turkish citizens who happen variously to sympathize with few, some or all of Gülen's teachings and, as normal citizens, are naturally distributed across workplaces in Turkey - including in academia, the military and government offices.

You also imply that the Gülen movement may have orchestrated the coup attempt on 15 July last year. However, sceptics like me are still waiting for an independent investigation into what happened that day and who was to blame. If some people have committed a crime, then it should be those individuals who pay the price, not the tens of thousands who have been sacked and/or imprisoned.

A. Mesut Erzurumluoglu University of Leicester, UK. ame26@le.ac.uk

\section{China's decadal pollution census}

China will conduct its second decadal pollution census in December 2017. This will provide a baseline for measuring the impact of environmental taxes that come into force next year, and for assessing discharge permits that will be issued for all pollution sources by 2020 . The data must therefore be reported with greater accuracy and more accountability than in the 2007 census.

That census targeted nearly 6 million industrial, agricultural and residential sources and centralized pollution-control facilities. However, large inconsistencies between the census data and those collected from other sources created big problems for the nationwide control system for total emissions. For instance, environmental statistics put the total discharge of organic pollutants in water at 15 million tonnes, whereas the census figure was double that.

Addressing these problems in the second census could prove difficult because China's economy and output have grown markedly over the past decade. Technologies such as big data, drones and remote-sensing satellites will help, backed by robust methodologies, overall transparency and public participation and supervision. Legal responsibility for falsifying pollution data or refusing to report pollution must also be clearly defined and enforced.

Qing Hu South University of Science and Technology of China, Shenzhen, China.

Xuetao Zhao Chinese Academy for Environmental Planning, Beijing, China.

Xiao Jin Yang Beijing University of Chemical Technology, China. yang.xj@outlook.com

\section{Shirts off to boost separation methods}

Shirts were a source of inspiration for starch-gel electrophoresis (E. Altschuler Nature 543, 179; 2017). They also figured in another pioneering separation technique - polyamide thin-layer chromatography.

At the National Taiwan University in 1958, KungTsung Wang and Yao-Tang Lin heard that German scientists were using polyamide - the primary constituent of nylon - for column chromatography. However, polyamide was unaffordable for scientists in Taiwan so soon after the Pacific War and Chinese Civil War.

The two decided to use their nylon shirts instead. They dissolved them in formic acid, extracted the nylon in ethanol, and air-dried it to a powder to act as a stationary-phase separation medium. Wang eventually published five Nature papers (1965-67) on the basis of results from polyamide thin-layer chromatography.

Min-Liang Wong National

Chung-Hsing University,

Taichung, Taiwan.

mlwong@dragon.nchu.edu.tw 